\title{
Electronic Structure Analysis of the Dinuclear Metal Center in the Bioremediator Glycerophosphodiesterase (GpdQ) from Enterobacter aerogenes
}

\author{
Kieran S. Hadler, ${ }^{\dagger}$ Nataša Mitić, ${ }^{\dagger}$ Sylvia Hsu-Chen Yip, ${ }^{\ddagger}$ Lawrence R Gahan, ${ }^{\dagger}$ David L. Ollis, ${ }^{\ddagger}$ \\ Gerhard Schenk, ${ }^{, \dagger}$ and James A. Larrabee ${ }^{\star, \S}$ \\ ${ }^{\dagger}$ School of Chemistry and Molecular Biosciences, The University of Queensland, St Lucia, Queensland, 4072, \\ Australia, ${ }^{\star}$ Research School of Chemistry, Australian National University, Canberra, ACT, 0200, Australia, and \\ ${ }^{\S}$ Department of Chemistry and Biochemistry, Middlebury College, Middlebury, Vermont 05753
}

Received October 2, 2009

\begin{abstract}
The glycerophosphodiesterase (GpdQ) from Enterobacter aerogenes is a promiscuous, dinuclear metallohydrolase that has potential application in the remediation of organophosphate nerve agents and pesticides. GpdQ employs an unusual reaction mechanism in which the enzyme is predominantly mononuclear in the resting state, and substrate binding induces the formation of the catalytically competent dinuclear center (Hadler et al. J. Am. Chem. Soc. 2008, $130,14129)$. Reactivity is further modulated by the coordination flexibility of Asn80, a ligand that binds to the second, loosely bound metal ion (Hadler et al. J. Am. Chem. Soc. 2009, 131, 11900). It is proposed that hydrolysis is initiated by a terminal, metal-bound hydroxide molecule which is activated at unusually low pH by electrostatic/hydrogen bonding interactions with a bridging hydroxide species. In this study, electronic structure analysis of the dinuclear center is employed to study the coordination environment of the dinuclear center at the resting and product-bound stage of catalysis. This is achieved through the use of variable temperature, variable field magnetic circular dichroism experiments involving the Co(II)-substituted wild type enzyme and its Asn80Asp variant. The data support the above model for the catalytic mechanism whereby the metal ion-bridging hydroxide molecule activates a terminally bound hydroxide nucleophile. Replacement of Asn80 by an aspartate residue does prevent coordination flexibility but also leads to cleavage of the $\mu$-hydroxide bridge and reduced reactivity. This is the first study to investigate the electronic structure of an enzyme with a $\mu-1,1$-carboxylate bridged dicobalt(II) center.
\end{abstract}

\section{Introduction}

Enterobacter aerogenes glycerophosphodiesterase (GpdQ) is a dinuclear metallohydrolase which has an unusually broad selectivity toward phosphate ester substrates. ${ }^{1-4}$ The ability of GpdQ to hydrolyze the $\mathrm{P}-\mathrm{O}$ bond of phosphate tri-, di-, and monoesters has attracted attention for its potential application in the bioremediation of organophosphate nerve agents and pesticides. ${ }^{5-7}$

*To whom correspondence should be addressed. E-mail: schenk@uq edu.au (G.S.), larrabee@middlebury.edu (J.A.L.).

(1) Mitić, N.; Smith, S. J.; Neves, A.; Guddat, L. W.; Gahan, L. R.; Schenk, G. Chem. Rev. 2006, 106, 3338-3363.

(2) Wilcox, D. E. Chem. Rev. 1996, 96, 2435-2458

(3) Jackson, C. J.; Carr, P. D.; Liu, J.-W.; Watt, S. J.; Beck, J. L.; Ollis, D. L. J. Mol. Biol. 2007, 367, 1047-1062.

(4) McLoughlin, S. Y.; Jackson, C.; Liu, J.-W.; Ollis, D. L. Appl. Environ. Microbiol. 2004, 70, 404-412.

(5) Ely, F.; Foo, J. L.; Jackson, C. J.; Gahan, L. R.; Ollis, D.; Schenk, G. Curr. Top. Biochem. Res. 2007, 9, 63-78.

(6) Ghanem, E.; Li, Y.; Xu, C.; Raushel, F. M. Biochemistry 2007, 46, 9032-9040.

(7) Although GpdQ can hydrolyze all three classes of phosphate esters, the diesterase activity is significantly higher than the mono- and triesterase activity. Efforts to increase the triesterase activity by directed evolution are currently in progress.
The active site structure of GpdQ comprises a strongly bound metal ion in the $\alpha$ site, coordinated by four amino acid residues (Asp8, His10, His197, and Asp50, the latter bridging the two metal ions), a terminal water ligand and a hydr(oxide) molecule that bridges the two metal ions (Figure 1). ${ }^{3,8,9}$ The metal ion in the $\beta$ site is loosely bound and is also coordinated by four amino acid residues (Asp50, Asn80, His156, and His195). Asn80 has been shown to play a critical role in regulating the reactivity of GpdQ. ${ }^{8,10}$ Spectroscopic studies of GpdQ mutants where this asparagine was replaced by alanine (no metal binding ability) or aspartate (enhanced metal binding ability) indicated that Asn 80 is important in controlling the metal ion coordination of GpdQ. ${ }^{8}$ In conjunction with steady-state kinetics and stopped-flow

(8) Hadler, K. S.; Tanifum, E. A.; Yip, S. H.-C.; Mitić, N.; Guddat, L. W.; Jackson, C. J.; Gahan, L. R.; Nguyen, K.; Carr, P. D.; Ollis, D. L.; Hengge, A. C.; Larrabee, J. A.; Schenk, G. J. Am. Chem. Soc. 2008, 130, 14129-14138.

(9) Hadler, K. S.; Huber, T.; Cassady, A. I.; Weber, J.; Robinson, J.; Burrows, A.; Kelly, G.; Guddat, L. W.; Hume, D. A.; Schenk, G.; Flanagan, J. U. BMC Res. Notes 2008, 1,78 .

(10) Hadler, K.; Mitić, N.; Ely, F.; Hanson, G. R.; Gahan, L. R.; Larrabee, J. A.; Ollis, D. L.; Schenk, G. J. Am. Chem. Soc. 2009, 131, 11900-11908. 


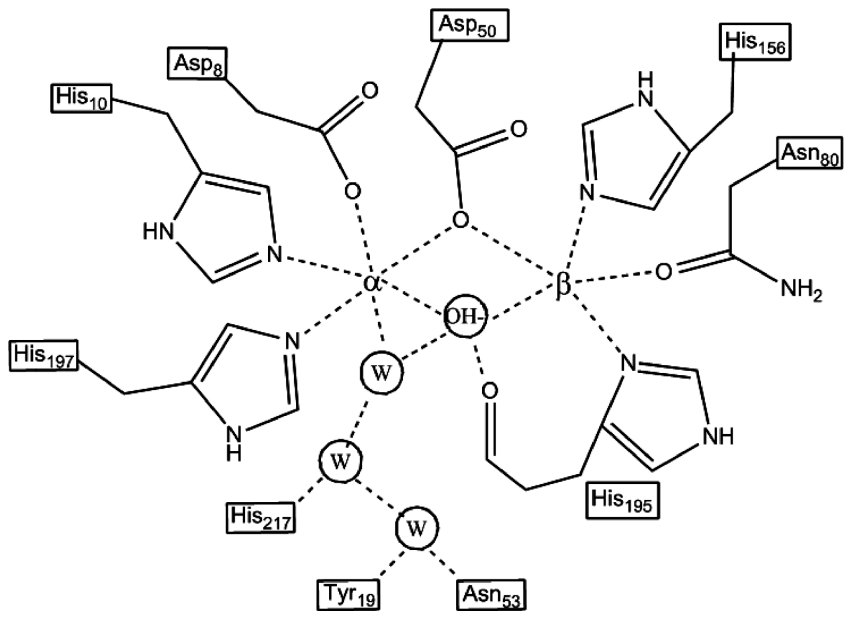

Figure 1. Schematic representation of the active site of wild type GpdQ.

fluorescence measurements, it was also found that the flexible coordination of Asn80 is an integral part of the reaction mechanism and is essential to achieve optimal reactivity. ${ }^{10} \mathrm{In}$ the Asn80Asp mutant where the degree of coordination flexibility is expected to be limited and the $\beta$ metal ion is bound tightly, the reactivity is significantly smaller than for the wild-type form of GpdQ. ${ }^{8}$ In contrast, in the Asn80Ala mutant the metal ion in the $\beta$ site only binds if substrate is present, and while the reactivity of this mutant is increased in comparison to wild-type GpdQ its affinity for substrate is greatly reduced. ${ }^{8}$ Hence, coordination flexibility is believed to assist optimal substrate binding and alignment throughout the catalytic cycle.

The in vivo metal ion composition of GpdQ is currently unclear (recombinantly expressed GpdQ coordinates Fe at the $\alpha$ site and some Fe and $\mathrm{Zn}$ at the weak binding $\beta$ site), ${ }^{11}$ but enzyme activity can be reconstituted in the presence of $\mathrm{Zn}(\mathrm{II}), \mathrm{Mn}(\mathrm{II})$, and $\mathrm{Co}(\mathrm{II}){ }^{3,4,10}$ The latter two metal ions facilitate active site structural studies using these metal ions' paramagnetic properties. Previous studies employed magnetic circular dichroism (MCD) of the $\mathrm{Co}(\mathrm{II})$-derivative of GpdQ as a qualitative means to examine the coordination environment (free enzyme, phosphate-bound enzyme and selected mutants), and electron paramagnetic resonance (EPR) of the $\mathrm{Mn}(\mathrm{II})$-derivative to assess the binding affinities of the metal ion in the $\alpha$ and $\beta$ sites. ${ }^{8,10}$ Combined with structural and kinetic data, a comprehensive catalytic mechanism has been proposed. ${ }^{8}$ In brief, GpdQ exists predominantly as a mononuclear enzyme in the resting state, and formation of the catalytically competent dinuclear center is induced by the substrate. Substrate binding to the $\beta$-metal ion induces the dissociation of Asn 80 and a hydroxide terminally bound to the $\alpha$ metal ion (activated via $\mathrm{H}$-bond interaction by the $\mu$-OH molecule) attacks the phosphorus atom of the substrate causing $\mathrm{P}-\mathrm{O}$ bond fission. The bound product molecule bridges the dinuclear center presumably in a $\mu-1,3$ mode, and the bridging hydroxide molecule is still intact. In the final stage, the product and $\beta$ metal ion are released, restoring the enzyme to the mononuclear resting state.

Although various species involved in this catalytic cycle have been spectroscopically observed, ${ }^{8}$ no information regarding the electronic structure of the metal ions has been

(11) Jackson, C. J.; Hadler, K. S.; Carr, P. D.; Oakley, A. J.; Yip, S.; Schenk, G.; Ollis, D. L. Acta Crystallogr. 2008, F62, 681-685. reported, and the role of the metal ion-bridging hydroxide is also still unclear. The Asn80Asp mutant of GpdQ is an ideal system to study both because of its high affinity for both metal ions. Here, we report a detailed analysis of the MCD and variable-temperature variable-field (VTVH) MCD properties of the Co(II)-derivative of both wild-type and mutant GpdQ. This study supports the proposed catalytic mechanism but also clarifies the roles of the bridging and terminal hydr(oxide) ligands in the hydrolytic reaction.

\section{Materials and Methods}

Preparation of Metal Ion Derivatives and Mutants of GpdQ. The $\mathrm{Co}_{2}$-GpdQ derivatives were prepared as previously described. ${ }^{8,10}$ The Asn80Asp mutation was introduced using the Stratagene QuikChange kit.

MCD Spectroscopy. Protein samples for MCD were dissolved in a $60 \% / 40 \%(\mathrm{v} / \mathrm{v})$ mixture of glycerol/buffer and used to fill a $0.62 \mathrm{~cm}$ path length brass sample cell with quartz windows. The buffer contained either $50 \mathrm{mM}$ HEPES (pH 8.0) or $50 \mathrm{mM}$ CHES (pH 10.0). The MCD system used has a JASCO J600 spectropolarimeter and an Oxford Instruments SM4000 cryostat/magnet. Data were collected at increments of $0.5 \mathrm{~T}$ from 0 to $7.0 \mathrm{~T}$ and at temperatures of 1.3, 3.0, 4.2, 10, 25, and $50 \mathrm{~K}$. Each spectrum was corrected for any natural CD by subtracting the zero-field spectrum of the sample. Even when there is no sample present the instrument baseline has a small deviation from zero ( $\pm 0-5$ millidegrees) that is both fieldand wavelength-dependent. Therefore, each spectrum was also corrected by subtraction of a spectrum recorded at the same magnetic field but with no sample present, which results in no deviation from zero when the magnetic field is zero. The resultant spectra were fitted to the minimum number of Gaussian peaks to achieve a satisfactory composite spectrum using the GRAMS AI software. The absolute values of the heights of each Gaussian peak were used in the VTVH MCD data analysis.

Angular Overlap Model Calculations. Angular overlap model (AOM) calculations were made using AOMX, a program developed and distributed by Adamsky and coworkers. ${ }^{12-14}$ AOMX determines the optimum ligand field parameters needed to fit an observed set of d-d transitions based on a given structure. Input to AOMX requires the ligand coordinates referred to the metal ion at the origin. The coordinates were generated from the published bond angles and distances (PDB accession code: 3D03). The Cartesian coordinates of the $\alpha$ and $\beta$ site ligands for each GpdQ species are shown in Supporting Information, Table S1. AOMX does not use the bond distances; only the angles are important, and these are fixed by the crystal structure. However, the bond distances are reflected by the magnitude of the resulting ligand field parameters, $\varepsilon_{\sigma}$ and $\varepsilon_{\pi}$. Each Co(II) metal site was treated separately.

The AOM is highly parametrized, and there is an insufficient number of observed d-d transitions to calculate

(12) Adamsky, H.; Schonherr, T.; Atanasov, M. In Comprehensive Coordination Chemistry II, Vol. 1.; Lever, A. B. P., Ed.; Elsevier: Oxford, 2004; pp 661-664.

(13) Available on the internet at http://www.aomx.de.

(14) Schonherr, T.; Atanasov, M.; Adamsky, H. In Comprehensive Coordination Chemistry II, Vol. 1.; Lever, A. B. P., Ed.; Elsevier: Oxford, 2004, pp 443-455. 
all the parameters (vide infra). However, the AOM handles low-symmetry situations very easily, so it is an excellent model to apply to these systems. By making the simplifying assumption of an average ligand field (i.e., no $\pi$-bonding and only one average $\varepsilon_{\sigma}$ ), relating the Racah parameters, $C$ to $B$ such that $C=4.6 B$, and using the actual structure around each metal ions based on available crystal structures, it is possible to use AOMX to demonstrate that the observed d-d transitions clearly fall into those arising from 6-coordinate $\mathrm{Co}(\mathrm{II})$ or 5-coordinate $\mathrm{Co}(\mathrm{II})$.

MCD Data Analysis. VTVH MCD data were normalized to the maximum observed intensity and were fitted using a Simplex algorithm that minimizes a chi-squared goodness of the fit parameter. A dimer model was used to fit VTVH MCD data of magnetically coupled Co(II)$\mathrm{Co}(\mathrm{II})$ systems. In the dimer model, wave functions are calculated using a spin Hamiltonian (Supporting Information, Equation S1), and the MCD intensity is given by the spin expectation values of the Co(II) ion responsible for the MCD transition. The dimer model yields the coupling constant, $J$; the single ion zero-field splitting parameters, $D$ and $E$; the polarization of the transition; and the energies and total $M_{s}$ values of the resulting wave functions. For two high-spin Co(II) ions, the dimer model yields 16 sublevels. The second fitting procedure uses a monomer model for isolated (uncoupled) $\mathrm{Co}$ (II) in which the MCD intensity is calculated identically to the dimer model except the spin Hamiltonian only includes one metal and does not include a $2 J \mathrm{~S}_{1} \cdot \mathrm{S}_{2}$ term. The models, theory, equations, and computer programs to fit the VTVH MCD data have been developed by the Solomon group over the past several years and are described in detail elsewhere. ${ }^{15-22}$ The computer programs and instructions for using them were kindly provided by the Solomon group. The VTVH MCD data sets that were fitted included all temperatures, nominally $1.3,3.0,4.2,10,25$, and $50 \mathrm{~K}$; however, only three isotherms (at 1.3, 4.2, and $10 \mathrm{~K}$ ) are shown in figures for clarity.

\section{Results}

MCD Spectra, VTVH Behavior, and Ligand Field Calculations. MCD spectra of wild type and mutant Asn80Asp-GpdQ containing 2 equiv of $\mathrm{Co}(\mathrm{II})$ have previously been reported. ${ }^{8}$ These spectra display two bands at $\sim 495 \mathrm{~nm}$ and $\sim 570 \mathrm{~nm}$, corresponding to six- and fivecoordinate $\mathrm{Co}$ (II) transitions, respectively (Table 1). Here, higher quality data are presented, allowing Gaussian deconvolution and analysis of VTVH MCD behavior. The transition energies calculated by AOM, based on

(15) Neese, F.; Solomon, E. I. Inorg. Chem. 1999, 38, 1847-1865.

(16) Reem, R. C.; Solomon, E. I. J. Am. Chem. Soc. 1987, 109, 1216-1226.

(17) Solomon, E. I.; Pavel, E. G.; Loeb, K. E.; Campochiaro, C. Coord. Chem. Rev. 1995, 144, 369-460.

(18) Strand, K. R.; Yang, Y. S.; Andersson, K.; Solomon, E. I. Biochemistry 2003, 42, 12223-12234.

(19) Wei, P. P.; Skulan, A. J.; Mitić, N.; Yang, Y. S.; Saleh, L.; Bollinger, J. M.; Solomon, E. I. J. Am. Chem. Soc. 2004, 126, 3777-3788.

(20) Wei, P. P.; Tomter, A. B.; Rohr, A. K.; Andersson, K.; Solomon, E. I. Biochemistry 2006, 45, 14043-14051.

(21) Mitić, N.; Clay, M. D.; Saleh, L.; Bollinger, J. M.; Solomon, E. I. J. Am. Chem. Soc. 2007, 129, 9049-9065.

(22) Mitić, N.; Saleh, L.; Schenk, G.; Bollinger, J. M.; Solomon, E. I. J. Am. Chem. Soc. 2003, 125, 11200-11201.
Table 1. Summary of Ligand Field Calculations ${ }^{a}$

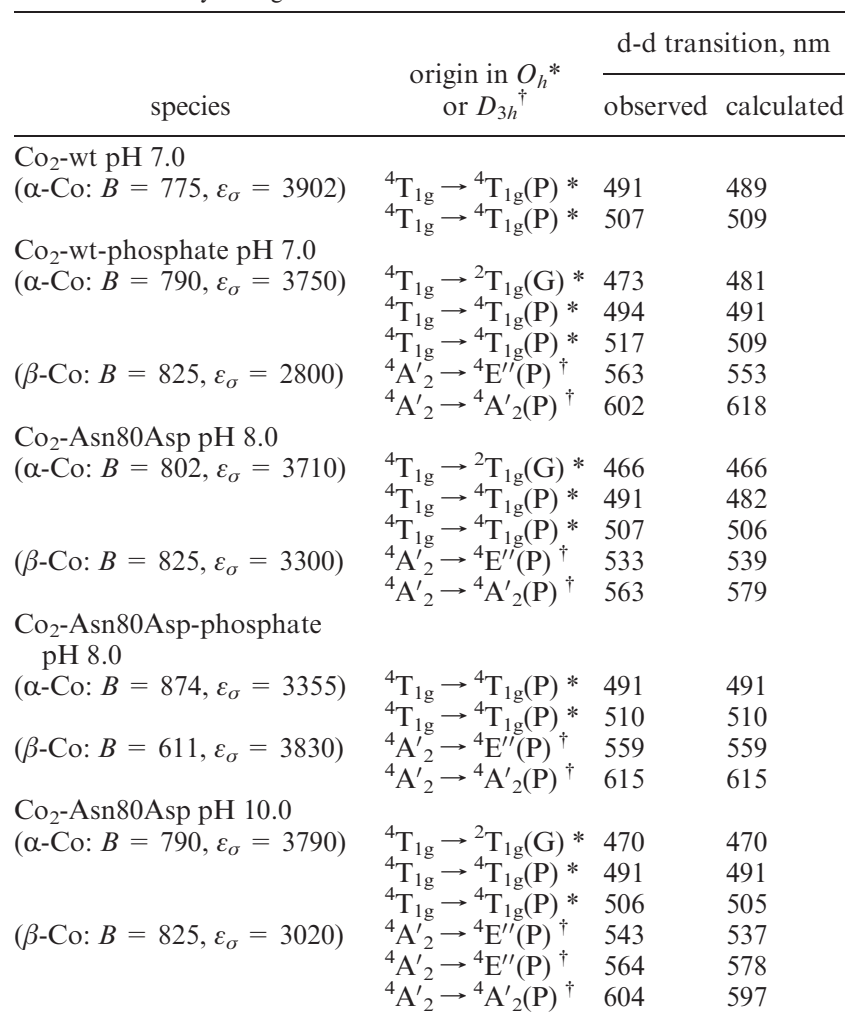

${ }^{a}$ These values correspond to transitions arising from the sixcoordinate $\alpha$ site $\left({ }^{*}\right)$ and five-coordinate $\beta$ site $\left(^{\dagger}\right)$. The Racah parameter $C$ was fitted according to $C=4.6 B$. The units for $B, C$ and $\varepsilon_{\alpha}$ are $\mathrm{cm}^{-1}$.

bond angles from the crystal structure, ${ }^{8}$ are compared to these experimental data. These calculations fit an average $\varepsilon_{\sigma}$ bonding parameter for all the ligands coordinated to the $\alpha\left\{\varepsilon_{\sigma}(\alpha)\right\}$ and $\beta\left\{\varepsilon_{\sigma}(\beta)\right\}$ sites, respectively. The general shapes and signs of the MCD transitions are qualitatively similar to previously reported MCD spectra for sixcoordinate and five-coordinate $\mathrm{Co}(\mathrm{II})$ complexes and $\mathrm{Co}(\mathrm{II})$-substituted enzymes. ${ }^{23,24}$ In particular a negative MCD peak near $500 \mathrm{~nm}$ followed by a weak positive peak to higher energy and is characteristic for six-coordinate $\mathrm{Co}(\mathrm{II})$. Other than these qualitative similarities, no other additional interpretation of the sign of the MCD transitions is possible without detailed information about the vibrations in the ground and excited state which enable these vibronic transitions. ${ }^{25}$

Co(II)-wt-GpdQ, pH 7.0. The MCD spectrum of the wild type enzyme with 2 equiv of $\mathrm{Co}(\mathrm{II})$ added (pH 7.0) has been previously reported. ${ }^{8} \mathrm{GpdQ}$ exists predominantly as a mononuclear enzyme in this state with the six-coordinate $\alpha$ site occupied and the $\beta$ site virtually unoccupied. The single MCD band can be resolved into two transitions at 491 and $507 \mathrm{~nm}$ (Figure 2). Ligand field calculations performed on the $\alpha$ metal ion binding site in the crystal structure accurately predict the energy of these transitions. The optimized AOMX parameters are shown in Table 1. The Racah parameters, $B$ and $\mathrm{C}$,

(23) Kaden, T. A.; Holmquist, B.; Vallee, B. L. Inorg. Chem. 1974, 13, 2585-2590.

(24) Kaden, T. A.; Holmquist, B.; Vallee, B. L. Biochem. Biophys. Res. Commun. 1972, 46, 1654-1659.

(25) Harding, M. J.; Briat, B. Mol. Phys. 1973, 25, 745-776. 

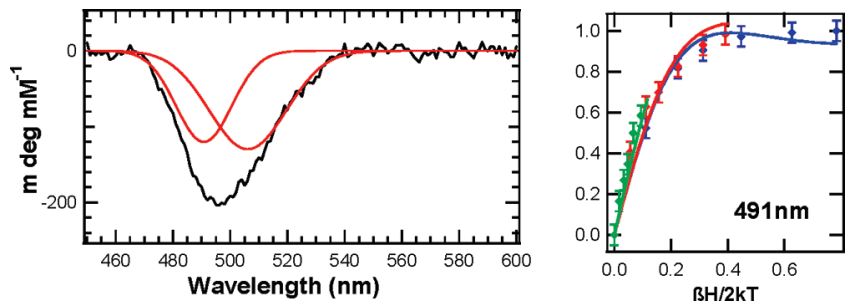

Figure 2. MCD spectrum (7 T) of wild type GpdQ at pH 7.0 (left) and fitted VTVH MCD data for the $491 \mathrm{~nm}$ transition (right). Temperatures of blue, red, green $(1.5,3.0,10.0 \mathrm{~K})$.

agree well with values obtained for other reported $\mathrm{Co}$ (II) complexes. $^{26}$

The magnetization curve of the $491 \mathrm{~nm}$ band is also shown in Figure 2. Uncoupled six-coordinate $\mathrm{Co}(\mathrm{II})$ experiences strong zero field splitting $\left(2 D>100 \mathrm{~cm}^{-1}\right)$ of the spin quartet ground state, splitting the ground state into two pseudo-Kramers doublets, separated by $>100 \mathrm{~cm}^{-1} .^{27}$ Therefore, the low temperature VTVH MCD data from isolated six-coordinate $\mathrm{Co}(\mathrm{II})$ can often be treated as an isolated pseudo-Kramers doublet. ${ }^{27}$ An indication that this is indeed the case here is the observation of nearly superimposed isotherms in the magnetization plots (Figure 2). The data were thus fitted to eq 1 for an isolated Kramers doublet. An excellent fit with $g=$ 7.1 was obtained (fit not shown). In this equation, $\Delta \varepsilon$ is the MCD intensity, $I$ is the intensity at saturation, $g$ is the isotropic effective $\mathrm{g}$ factor, $\beta$ is the Bohr magneton, $\mathrm{H}$ is the magnetic field, $k$ is Boltzmann's constant, $T$ is the absolute temperature, and $B$ is the temperatureindependent $\mathrm{B}$ term intensity. ${ }^{28}$

$$
\Delta \varepsilon=I \tanh \left(\frac{g \beta \mathrm{H}}{2 k T}\right)+B \mathrm{H}
$$

The data were also fitted using the monomer model (i.e., using a spin Hamiltonian for an isolated $\mathrm{Co}$ (II) center). A satisfactory fit, shown in Figure 2, was obtained with the parameters listed in Table 2.

Co(II)-wt-GpdQ-phosphate Complex, pH 7.0. The MCD spectrum shown in Figure 3 arises when 20 equiv of phosphate are added to the previous sample (wild type $\mathrm{GpdQ}$ with 2 equiv of $\mathrm{CoCl}_{2}$ ). The bands are resolved into five transitions at 473, 494, 517, 563, and $602 \mathrm{~nm}$, respectively. In addition to the six-coordinate $\mathrm{Co}(\mathrm{II})$ transitions at 494 and $517 \mathrm{~nm}$ seen at similar energy in the uncomplexed species, a weaker positive doublet transition at $473 \mathrm{~nm}$ is also attributable to six-coordinate Co(II). ${ }^{25}$ The bands at 563 and $602 \mathrm{~nm}$ are due to fivecoordinate $\mathrm{Co}(\mathrm{II})$ in the $\beta$ site. ${ }^{8}$ No phosphate-bound structure of GpdQ exists from which bond angle information can be used to perform ligand field calculations. However, previous studies suggest that phosphate binds in a $\mu-1,3$ manner to both $\mathrm{Co}(\mathrm{II})$ ions (this proposal is also supported by this work, vide infra). To approximate the

(26) Larrabee, J. A.; Alessi, C. M.; Asiedu, E. T.; Cook, J. O.; Hoerning, K. R.; Klingler, L. J.; Okin, G. S.; Santee, S. G.; Volkert, T. L. J. Am. Chem. Soc. 1997, 119, 4182-4196.

(27) Larrabee, J. A.; Leung, C. H.; Moore, R. L.; Thamrong-Nawasawat, T.; Wessler, B. S. H. J. Am. Chem. Soc. 2004, 126, 12316-12324.

(28) Johnson, M. K. In Physical Methods in Bioinorganic Chemistry; Que, L., Ed.; University Science: Sausalito, CA, 2000; p 245. geometry of GpdQ in this state, a structural alignment was performed between the crystal structures of the resting state GpdQ enzyme and a $\mu$-1,3-phosphate complex of Rv0805, a closely related enzyme with an identical active site, as shown in Supporting Information, Figure $\mathrm{S} 1$. The Asn80 ligand was ignored since it is known to dissociate upon phosphate binding. ${ }^{8}$ The calculations agree reasonably well with the assignment of these transitions to the $\alpha$ and $\beta \mathrm{Co}$ (II) ions (Table 1). The optimized AOMX parameters are shown in Table 1 .

The resolution of the five-coordinate band at $563 \mathrm{~nm}$ from the six-coordinate band at 517 is poor, and the signal/noise ratio is not very good in the MCD spectra of this sample, particularly at higher temperatures and lower magnetic fields strengths. Consequently, the VTVH MCD data for the five-coordinate $\mathrm{Co}$ (II) could not be accurately fitted. However, the VTVH MCD data for the $494 \mathrm{~nm}$ transition are of good quality, and the corresponding magnetization curve is shown in Figure 3. The addition of phosphate has led to a change in the VTVH MCD behavior, illustrated by the appearance of nested isotherms, an indication that the electronic ground state of the six-coordinate $\mathrm{Co}(\mathrm{II})$ in the $\alpha$ site has changed when the $\beta$ site becomes occupied by $\mathrm{Co}(\mathrm{II})$. The most plausible explanation for the change in the ground state properties is that the two Co(II) ions are magnetically exchanged-coupled through the hydr(oxide) and $\mu$-1,1carboxylate bridging ligands (Figure 1). The electronic ground state of the six-coordinate $\mathrm{Co}(\mathrm{II})$ is converted from an isolated pseudo Kramers doublet to one in which there are a manifold of 16 sublevels, several of which can be populated at low temperatures. Accordingly, the magnetization data were fitted to the dimer model. The best fit parameters are listed in Table 2, and the fit is shown in Figure 3. The fit was not significantly improved by floating the rhombic ZFS parameter, $E$, for either metal ion, and hence these were set to zero. The fit was also not sensitive to the axial ZFS parameter, $D$, for the sixcoordinate $\mathrm{Co}(\mathrm{II})$ as long as it was greater than $50 \mathrm{~cm}^{-1}$, but it was very sensitive to $D$ for the five-coordinate $\mathrm{Co}(\mathrm{II})$ and to the coupling constant, $J$. The value of $D=3.5 \mathrm{~cm}^{-1}$ is typical of five-coordinate $\mathrm{Co}(\mathrm{II}){ }^{26,27,29}$ The Co(II) ions are weakly ferromagnetically coupled with $J=4.7 \mathrm{~cm}^{-1}$.

Co(II)-Asn80Asp-GpdQ, pH 8.0. The MCD spectrum of Asn80Asp-GpdQ with 2 equiv of $\mathrm{Co}(\mathrm{II})$ at $\mathrm{pH} 8.0$ is shown in Figure 4. The spectrum could be fitted with a minimum of five Gaussians: 466, 491, and 507 (sixcoordinate transitions), as well as 533 and $563 \mathrm{~nm}$ for the five-coordinate transitions. On the basis of geometric information from the crystal structure of $\mathrm{Co}(\mathrm{II})-\mathrm{GpdQ}$ (3D03), ligand field calculations were performed to predict the transition energies arising from each $\mathrm{Co}(\mathrm{II})$ ion (Table 1). The calculated values agree reasonably with the observed transitions for the six- $(\alpha)$ and five-coordinate $(\beta) \mathrm{Co}(\mathrm{II})$ species (Table 1$)$. The optimized AOMX parameters are shown in Table 1.

The magnetization plots for the transitions at 491 and $563 \mathrm{~nm}$ are also shown in Figure 4. The isotherms for the six coordinate transition at $491 \mathrm{~nm}$ are slightly nested

(29) Larrabee, J. A.; Johnson, W. R.; Volwiler, A. S. Inorg. Chem. 2009, $48,8822-8829$. 
Table 2. Best Fit Electronic Parameters of GpdQ Measured by VTVH MCD ${ }^{a}$

\begin{tabular}{|c|c|c|c|c|c|c|c|c|}
\hline \multirow[b]{2}{*}{ parameter } & \multirow{2}{*}{$\frac{\mathrm{Co}_{2}-\mathrm{wt} \mathrm{pH} 7.0}{491 \mathrm{~nm}}$} & \multirow{2}{*}{$\begin{array}{c}\mathrm{Co}_{2} \text {-wt-phosphate } \\
\text { pH } 7.0\end{array}$} & \multicolumn{2}{|c|}{$\begin{array}{c}\mathrm{Co}_{2}-\mathrm{Asn} 80 \mathrm{Asp} \\
\mathrm{pH} 8.0\end{array}$} & \multicolumn{2}{|c|}{$\begin{array}{l}\mathrm{Co}_{2} \text {-Asn } 80 \mathrm{Asp}- \\
\text { phosphate pH } 8.0\end{array}$} & \multicolumn{2}{|c|}{$\begin{array}{c}\mathrm{Co}_{2}-\mathrm{Asn} 80 \mathrm{Asp} \text { (with and } \\
\text { without phosphate) pH } 10.0\end{array}$} \\
\hline & & & $491 \mathrm{~nm}$ & $567 \mathrm{~nm}$ & $491 \mathrm{~nm}$ & $559 \mathrm{~nm}$ & $491 \mathrm{~nm}$ & $564 \mathrm{~nm}$ \\
\hline$J$ (between $\alpha$ and $\beta$ metal ions) $\left(\mathrm{cm}^{-1}\right)$ & & 4.7 & 1.2 & 1.1 & 4.7 & 5.8 & 10.1 & 10.0 \\
\hline$D(\alpha$ metal $)\left(\mathrm{cm}^{-1}\right)$ & $\geq 50$ & $\geq 50$ & $\geq 50$ & $\geq 50$ & $\geq 50$ & $\geq 50$ & $\geq 50$ & $\geq 50$ \\
\hline$D(\beta$ metal $)\left(\mathrm{cm}^{-1}\right)$ & & 3.5 & 6.9 & 6.9 & 5.3 & 6.4 & -4.6 & -4.4 \\
\hline$E(\alpha, \beta$ metal $)\left(\mathrm{cm}^{-1}\right)^{b}$ & & 0 & 0 & 0 & 0 & 0 & 0 & 0 \\
\hline$M_{x} *$ & $32 \%$ & $38 \%$ & $66 \%$ & $77 \%$ & $38 \%$ & $32 \%$ & $38 \%$ & $79 \%$ \\
\hline$M_{y} *$ & $66 \%$ & $56 \%$ & $16 \%$ & $15 \%$ & $56 \%$ & $62 \%$ & $56 \%$ & $18 \%$ \\
\hline$M_{z} *$ & $2 \%$ & $6 \%$ & $18 \%$ & $8 \%$ & $6 \%$ & $6 \%$ & $5 \%$ & $3 \%$ \\
\hline
\end{tabular}

$a *$ Calculated from $M_{x y}, M_{y z}$ and $M_{x z}$ using $\% M_{x}=\left(M_{x y} \bullet M_{y z}\right)^{2} /\left[\left(M_{x y} \bullet M_{x z}\right)^{2}+\left(M_{x y} \bullet M_{y z}\right)^{2}+\left(M_{x} \bullet M_{y z}\right)^{2}\right] . M_{y}$ and $M_{z}$ were calculated correspondingly. ${ }^{38}$ The fitting program grossly underestimated the errors in $J$ and $D$. We approximate these errors to be $\pm 15 \%$. ${ }^{b} E$ was allowed to float in the initial fits. The fits were highly insensitive to $E$, but when floated, gave very small values $(<0.05)$. Thus $E$ was set to zero for the final fit.
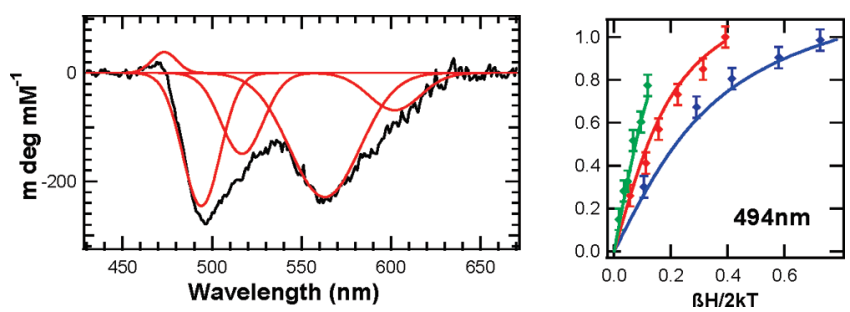

Figure 3. MCD spectrum (7 T) of wild type GpdQ-phosphate complex at $\mathrm{pH} 7.0$ (left) and fitted VTVH MCD data for the $494 \mathrm{~nm}$ transition (right). Temperatures of blue, red, green $(1.6,3.0,10.0 \mathrm{~K})$.
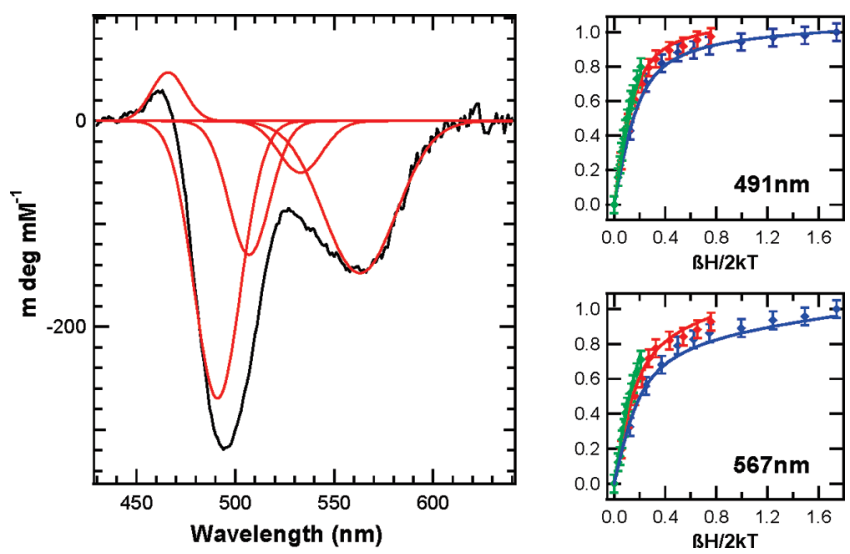

Figure 4. MCD spectrum (7 T) of Asn80Asp GpdQ at pH 8.0 (left) and fitted VTVH MCD data for the $491 \mathrm{~nm}$ and $567 \mathrm{~nm}$ transitions (right). Temperatures of blue, red, green $(1.4,3.1,11.3 \mathrm{~K})$.

indicating that there may be a manifold of low-lying excited states, which arise from an exchange interaction with the five-coordinate $\mathrm{Co}(\mathrm{II})$ ion (the nesting behavior becomes more obvious at higher $\mathrm{pH}$; vide infra). The magnetization data were fitted for each peak using the dimer model independently (Figure 4), and the parameters (Table 2) agree well regardless of whether they were derived from the six-coordinate or the five-coordinate VTVH MCD data fitting. The Co(II) ions are more weakly ferromagnetically coupled than in the wild type enzyme with added phosphate $\left(J=1.1\right.$ to $\left.1.2 \mathrm{~cm}^{-1}\right)$. There is also a small increase in the ZFS of the $\beta$ metal ion, consistent with a ligand change and/or a change in a ligand-metal-ligand angle.

Co(II)-Asn80Asp-GpdQ-phosphate Complex, pH 8.0. The MCD spectrum of Asn80Asp-GpdQ at $\mathrm{pH} 8.0$ in complex with phosphate is shown in Figure 5. The addition of phosphate leads to fairly dramatic changes in the
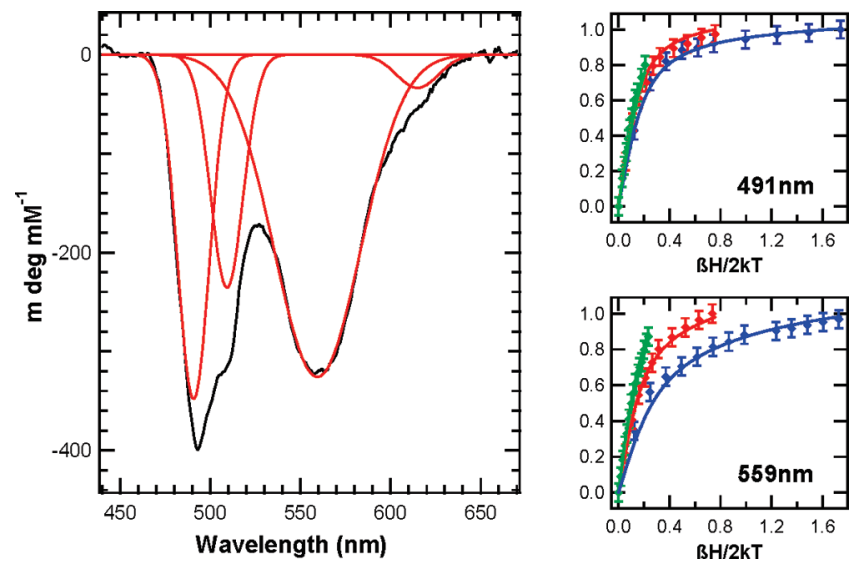

Figure 5. MCD spectrum (7 T) of the Asn80Asp-phosphate complex of GpdQ at pH 8.0 (left) and fitted VTVH MCD data for the $491 \mathrm{~nm}$ and 559 $\mathrm{nm}$ transitions (right). Temperatures of blue, red, green $(1.4,3.2,10.1 \mathrm{~K})$.

shape of both the six- and the five-coordinate $\mathrm{Co}$ (II) bands including (i) the disappearance of the positive transition at $471 \mathrm{~nm}$, (ii) the emergence of a distinct shoulder at $508 \mathrm{~nm}$, and (iii) a shift in the position of the five-coordinate bands to shorter wavelength (Figure 5). A fit to the minimum number of Gaussians gave transitions at 491, 510, 559, and $602 \mathrm{~nm}$. Ligand field calculations were performed using the alignment between the Rv0805-phosphate structure and GpdQ, as described above. The bridging hydr(oxide) ligand was not included in calculations for the $\beta$-metal ion as this bond is likely to be disrupted by the binding of phosphate to the Asn80Asp mutant (vide infra). There is a good agreement between the experimental and calculated d-d transition energies (Table 1). The optimized AOMX parameters are shown in Table 1.

The VTVH MCD behavior of the 491 and $559 \mathrm{~nm}$ transitions are shown in Figure 4. In comparison to free Asn80Asp GpdQ at identical pH (Figure 4) there is a greater degree of nesting in the magnetization behavior after the addition of phosphate, especially for the lower energy band (Figure 5). The data were fitted using the dimer model. The best fit to the data (Figure 5) reveals an increase in the ferromagnetic exchange coupling $(J=$ $4.7-5.8 \mathrm{~cm}^{-1}$ ) and small changes in the ZFS of the $\beta \mathrm{Co}(\mathrm{II})$ ion (Table 2).

Co(II)-Asn80Asp-GpdQ, pH 10.0 (with and without Phosphate). The MCD spectrum of Asn80Asp-GpdQ at $\mathrm{pH} 10.0$ is shown in Figure 6. It is virtually identical to the 

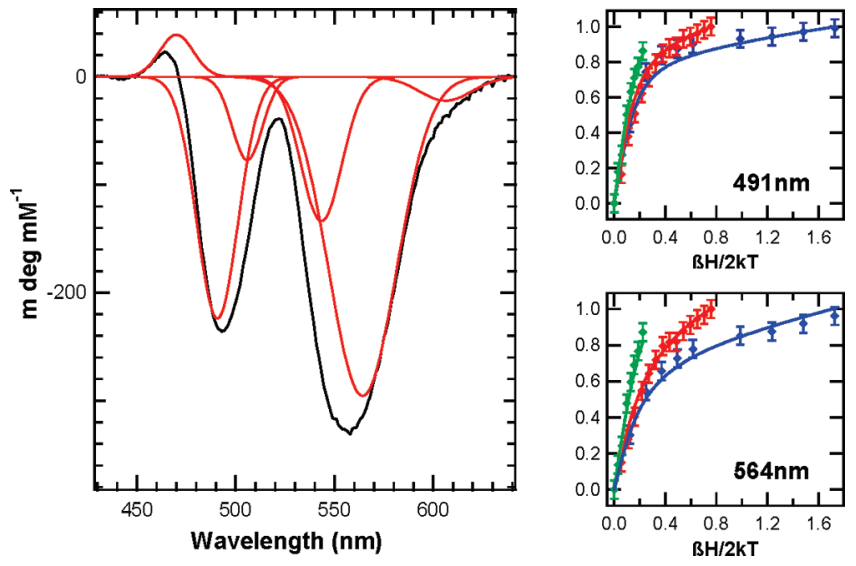

Figure 6. MCD spectrum (7 T) of Asn80Asp at pH 10.0 (left) and fitted VTVH MCD data for the $491 \mathrm{~nm}$ and $564 \mathrm{~nm}$ transitions (right). The spectrum and VTVH MCD behavior are unchanged when phosphate is added. Temperatures of blue, red, green $(1.4,3.1,10.4 \mathrm{~K})$.

MCD spectrum of Asn80Asp-GpdQ at $\mathrm{pH} 10.0$ in the presence of phosphate (Supporting Information, Figure S2). Inhibition studies of GpdQ with phosphate have shown that the $K_{\mathrm{i}}$ increases dramatically with $\mathrm{pH}$ and, at $\mathrm{pH} 10.0$, phosphate has practically no effect. ${ }^{8}$ The identical MCD spectra at $\mathrm{pH} 10.0$ with and without phosphate thus confirm that phosphate is probably not bound to the active site at this $\mathrm{pH}$. Six Gaussians were required to fit the data to give transitions at 470, 491, 506, 543,564 , and $604 \mathrm{~nm}$ (Figure 6). Ligand field calculations using the same structural information as for Asn80AspGpdQ at $\mathrm{pH} 8.0$ gave close agreement between experimental and calculated transition energies (Table 1). The optimized AOMX parameters are shown in Table 1.

The magnetizations plots of the 491 and $564 \mathrm{~nm}$ transitions are also shown in Figure 6. The strongly nested behavior in both sets of data reflect a significant change in the ground state in comparison to both the free and the phosphate-bound $\mathrm{Co}_{2}-\mathrm{Asn} 80 \mathrm{Asp}-\mathrm{GpdQ}$ species at $\mathrm{pH}$ 8.0. The data were fitted using the dimer model, and the parameters obtained from the best fit give $J=$ $10.0-10.1 \mathrm{~cm}^{-1}$ and a change in sign of the ZFS of the $\beta \operatorname{Co}(\mathrm{II})$ ion $\left(D=-4.4\right.$ to $\left.-4.6 \mathrm{~cm}^{-1}\right)$ (Table 2$)$.

\section{Discussion}

X-ray crystallography and MCD spectroscopy have been used in previous studies to examine the coordination environment of the metal ions and its relevance to catalysis. Structures of various catalytic stages including the resting state and product-bound species have been proposed. ${ }^{8}$ On the basis of these structures in conjunction with other kinetic and spectroscopic data, a plausible reaction mechanism has been reported. ${ }^{8,10,30}$ Here, a comprehensive analysis of the geometric and electronic structure of the dinuclear center is examined using the Co(II)-substituted derivative of the enzyme. This information is then used to verify aspects of the existing model for the catalytic mechanism.

Electronic Structure of GpdQ. MCD has recently proven to be a highly informative technique for examining

(30) Hadler, K. S.; Gahan, L. R.; Ollis, D. L.; Schenk, G. J. Inorg. Biochem. 2010, 104, 211-213.

(31) Larrabee, J. A.; Chyun, S. A.; Volwiler, A. S. Inorg. Chem. 2008, 47, 10499-10508.
Co(II)-containing enzymes ${ }^{27,31}$ and model systems, ${ }^{29,32,33}$ and is employed here to investigate the active site structure of GpdQ in detail. This technique was previously used to determine the solution structure of the two Co(II) ions in GpdQ by comparison with other known systems in the literature; in particular $\mathrm{Co}$ (II)-substituted methionine aminopeptidase (MetAP), a binuclear metallohydrolase with a mixed six-, five-coordination active site structure. ${ }^{27}$ This system gives rise to two MCD bands at similar wavelengths to that of GpdQ, and ligand field calculations as well as a VTVH MCD analysis have supported the assignment of the higher energy $(495 \mathrm{~nm})$ and lower energy $(567 \mathrm{~nm})$ bands to the six- and five-coordinate sites, respectively. However, recent studies of a mixed six-, five-coordinate model complex indicate that five-coordinate $\mathrm{Co}(\mathrm{II}) \mathrm{d}-\mathrm{d}$ transitions, depending on the geometry, can range from 471 to $638 \mathrm{~nm} .{ }^{29}$ Likewise, intense transitions due to a six-coordinate $\mathrm{Co}(\mathrm{II})$ species have been observed as high as $535 \mathrm{~nm}$ (a $588 \mathrm{~nm}$ transition has been observed from six-coordinate $\mathrm{Co}(\mathrm{II})$; however, this is a much weaker ${ }^{4} \mathrm{~T}_{1 \mathrm{~g}} \rightarrow{ }^{4} \mathrm{~A}_{2 \mathrm{~g}}$ transition). ${ }^{31}$

To clarify the previous assignment of the Co(II) transitions of GpdQ, we performed ligand field calculations and measured the axial ZFS of the Co(II) ion by VTVH MCD analysis. The ZFS is an excellent indicator of the coordination number for high spin $\mathrm{Co}(\mathrm{II})$, particularly for six-coordinate species in which the ZFS is extremely large $\left(D \geq 50 \mathrm{~cm}^{-1}\right)$, thus creating an effective pseudoKramers doublet ground state in isolated six-coordinate $\mathrm{Co}(\mathrm{II}){ }^{27}$ The resting state wild type enzyme has only one $\mathrm{Co}(\mathrm{II})$ ion bound (in the $\alpha$ site; Figure 1) and exhibits two transitions at 491 and $507 \mathrm{~nm}$ (Figure 2). The VTVH MCD isotherms are virtually overlaid, and fit to a model for an isolated $\mathrm{Co}(\mathrm{II})$ ion with a ZFS of $D \geq$ $50 \mathrm{~cm}^{-1}$ (Table 2), typical for uncoupled six-coordinate $\mathrm{Co}(\mathrm{II}){ }^{26,27,31,34}$ Almost identical transitions have been reported for six-coordinate $\mathrm{Co}(\mathrm{II})$ in MetAP and in related systems, and ligand field calculations support this assignment (Table 1).29

The binding of phosphate perturbs the six-coordinate $\alpha$ site by inducing a red-shift in the two Co(II) transitions and causing the appearance of a small positive transition at $473 \mathrm{~nm}$, which has previously been characterized as a six-coordinate $\mathrm{Co}(\mathrm{II})$ transition in MetAP ${ }^{27}$ and other cobalt complexes ${ }^{24}$ (Figure 3 ). The transition at $517 \mathrm{~nm}$ is similar in position to other known six- and five-coordinate $\mathrm{Co}(\mathrm{II})$ transitions and could thus potentially arise from either of the two cobalt ions. However, a comparison suggests that this band is equivalent to the $507 \mathrm{~nm}$ band of the mononuclear Co(II) species (Figure 2), thus having experienced a red-shift by $10 \mathrm{~nm}$ (Figure 3). The band at $\sim 563 \mathrm{~nm}$ is attributed to $\mathrm{Co}(\mathrm{II})$ in the $\beta$ site and can be resolved into two transitions at 563 and $602 \mathrm{~nm}$. The VTVH MCD behavior of the $563 \mathrm{~nm}$ band could not

(32) Johansson, F. B.; Bond, A. D.; Nielsen, U. G.; Moubaraki, B.; Murray, K. S.; Berry, K. J.; Larrabee, J. A.; McKenzie, C. J. Inorg. Chem. 2008, 47, 5079-5092.

(33) Xavier, F. R.; Neves, A.; Casellato, A.; Peralta, R. A.; Bortoluzzi, A. J.; Szpoganicz, B.; Severino, P. C.; Terenzi, H.; Tomkowicz, Z.; Ostrovsky, S.; Haase, W.; Ozarowski, A.; Krzystek, J.; Telser, J.; Schenk, G.; Gahan, L. R. Inorg. Chem. 2009, 48, 7905-7921.

(34) Krzystek, J.; A., Z. S.; Ozarowski, A.; Fiedler, A. T.; Brunold, T. C.; Telser, J. J. Am. Chem. Soc. 2004, 126, 2148-2155. 
be extracted; however, since the metal ions are coupled, the fit of the $494 \mathrm{~nm}$ data is dependent on the ZFS of both metal ions. The ZFS parameter $(D)$ was found to be $>50$ and $3.5 \mathrm{~cm}^{-1}$ for the $\alpha(494 \mathrm{~nm})$ and $\beta(563 \mathrm{~nm}) \mathrm{Co}(\mathrm{II})$ ions, respectively, consistent with six- and five-coordinate $\mathrm{Co}(\mathrm{II})$ in the $\alpha$ and $\beta$ sites, respectively (vide supra). ${ }^{8}$ Ligand field calculations support the assignment of these transitions, including the transition at $517 \mathrm{~nm}$ to the $\mathrm{Co}(\mathrm{II})$ ion in the $\alpha$ site (Table 1).

The spectrum of the resting state Asn80Asp mutant (Figure 4) reveals six-coordinate $\mathrm{Co}(\mathrm{II}) \mathrm{d}-\mathrm{d}$ transitions similar to those observed for the wild type enzyme (Figure 2). Additional lower energy d-d transitions arising from the now tighter bound $\mathrm{Co}(\mathrm{II})$ ion in the $\beta$ site are also observed. This spectrum is also consistent with that of the wild type enzyme where the low affinity $\beta$ metal ion site has been saturated with Co(II) by addition of a large excess of the metal ion salt. ${ }^{8}$ The binding of phosphate to the wild type enzyme results in the dissociation of the Asn 80 bond from the $\beta$ metal ion, forming a mixed six- $(\alpha)$, five-coordinate $(\beta)$ dicobalt(II) complex. ${ }^{8,10}$ However, in the Asn80Asp mutant, geometric changes induced by phosphate binding are not expected to be sufficient to break the Co(II)-Asp80 bond since aspartate is a stronger ligand than asparagine. Thus, a six-coordinate species in the $\beta$ metal ion site is anticipated when phosphate is added to the mutant enzyme. However, the MCD spectra (Figure 5) in conjunction with ligand field calculations (Table 1) and VTVH MCD analysis $\left(D_{\beta}=\right.$ $5.3-6.4 \mathrm{~cm}^{-1}$; Table 2) indicate that the $\beta$ metal ion is again five-coordinate, similar to wild type GpdQ. A likely explanation for this observation is that the Asp80 ligand, through a trans effect type mechanism $\left(\angle \mathrm{O}-\mathrm{CO}-\mathrm{O}=153^{\circ}\right)$ causes the breakage of the bond between the $\mu-\mathrm{OH}$ ligand and the $\beta$ metal ion (Figure 1). This would account for the following: (i) spectral differences between both the six- and five-coordinate $\mathrm{Co}(\mathrm{II})$ MCD bands of the phosphate bound wild type (Figure 3) and Asn 80 Asp mutant (e.g., the emergence of a distinct shoulder in Asn80Asp; Figure 5); (ii) a modest change in the ZFS of the $\beta$ site in the mutant as compared to the wild type enzyme (since both Asp80 and $\mu-\mathrm{OH}$ are equatorial ligands of a trigonal based pyramid, a significant change would not be expected; ${ }^{3}$ Table 2); (iii) a slightly weaker ligand field around the $\beta$ metal (replacing a bond to the $\mu$-OH ligand with a bond to phosphate); and (iv) may contribute to the significantly reduced catalytic activity of the Asn80Asp mutant since the $\mu-\mathrm{OH}$ is proposed to activate the terminally bound nucleophile in catalysis. ${ }^{8}$

Significant spectral changes also occur upon increasing the $\mathrm{pH}$ from 8 to 10 (Figure 6). The most marked change is the intensification of the five-coordinate $\beta \mathrm{Co}(\mathrm{II})$ transitions, relative to that of the $\alpha \mathrm{Co}$ (II). The positions of the six-coordinate transitions at $470 \mathrm{~nm}, 491$ and $506 \mathrm{~nm}$ are almost identical; however, the shape of the five-coordinate band is somewhat altered and requires a minimum of three Gaussians (543 nm, 564 and $604 \mathrm{~nm}$; Figure 6). While the magnitude of the ZFS again supports the presence of six- and five-coordinate Co(II) species, the sign of the ZFS of the five-coordinate $\beta \mathrm{Co}$ (II) ion has changed (Table 2), indicating that a considerable geometric rearrangement occurs as the $\mathrm{pH}$ is increased.
Such a change in ZFS is likely to be associated with a compression in the angle between the two axial ligands of the trigonal pyramidal $\beta$ site (His195 and the $\mu-1,1-$ carboxylate species of Asp50; Figure 1). ${ }^{3}$ A 10-fold increase in the exchange coupling from $\mathrm{pH} 8$ to 10 (Table 2) suggests that this rearrangement is coupled with a removal of a proton from a bridging group, in this case the deprotonation of $\mu$-hydroxide to $\mu$-oxide (see below). According to the crystal structure of GpdQ the $\mu$-hydroxide group forms a hydrogen bond with the backbone carbonyl oxygen of His195 (Figure 1). Thus, as the $\mathrm{pH}$ is increased from 8 to 10 , and the $\mu$-hydroxide is deprotonated, the disruption of this hydrogen bonding interaction is mediating the required geometric changes that lead to the observed change in the sign of ZFS.

Metal Ion Interactions. The catalytic mechanism of GpdQ is believed to involve a nucleophilic attack by a hydroxide molecule bound to the $\alpha$ metal ion, which has been activated via hydrogen bonding by the bridging hydroxide molecule (Figure 1). ${ }^{8}$ The observation (vide supra) that the bridging hydroxide is deprotonated to a $\mu$-oxide species at $\mathrm{pH} 10.0$ is consistent with its functionality only as a nucleophile-activator, not a nucleophile itself, since no significant changes in kinetic activity are observed at this $\mathrm{pH}^{8}$ Subsequent to a nucleophilic attack, the bridging hydroxide molecule is believed to remain intact in wild type $\mathrm{GpdQ}^{8}$ and displaced to a terminal position on the $\alpha$ metal ion in the Asn80Asp mutant (vide supra). To investigate changes in the bridging ligand(s), the exchange coupling was investigated through VTVH MCD analysis. The vast majority of studies that investigate the electronic structure of dinuclear $\mathrm{Co}(\mathrm{II})$ systems have focused on $\mu$-1,3-carboxylate bridged centers. ${ }^{29,31,32,35}$ Since these ligands are very poor mediators of exchange coupling, ${ }^{32}$ the coupling in these cases can be considered to arise predominantly from the bridging water molecule. On the basis of the studies of these systems, a trend has emerged which suggests that ferromagnetic coupling occurs via $\mu$-hydroxo bridges and no coupling or weak antiferromagnetic coupling occurs via $\mu$-aqua bridges. ${ }^{29,31,32,35}$ Only a single model complex with a $\mu$-1,1-carboxylate bridge similar to GpdQ has been reported. This complex has no bridging water molecule and has ferromagnetic coupling of $18 \mathrm{~cm}^{-1} .36$

In the resting state of GpdQ, exchange coupling is potentially mediated through both the $\mu-1,1$ aspartate ligand and the bridging water molecule. Because of the large excess of metal ions required to form the binuclear metal ion center, the coupling in the wild type enzyme cannot be measured in the absence of substrate/inhibitor. However, for the Asn80Asp enzyme at $\mathrm{pH}$ 8.0, the cobalt ions exhibit a weak ferromagnetic coupling (Table 2), supportive of the bridging water existing as a $\mu-\mathrm{OH}$ species. At $\mathrm{pH} 10.0$, a 10 -fold increase in exchange coupling is observed (Table 2). Since the bridging water molecule is unusually acidic ${ }^{8}\left(\mathrm{p} K_{\mathrm{a}} \sim 3.2\right.$ for $\mu-\mathrm{H}_{2} \mathrm{O}$ to $\mu-\mathrm{OH})$, it is likely that the observed increase in the

(35) Brown, D. A.; Errington, W.; Glass, W. K.; Haase, W.; Kemp, T. J.; Nimir, H.; Ostrovsky, S. M.; Werner, R. Inorg. Chem. 2001, 40, 5962-5971.

(36) Ostrovsky, S. M.; Falk, K.; Pelikan, J.; Brown, D. A.; Tomkowicz, Z.; Haase, W. Inorg. Chem. 2006, 45, 688-694. 
coupling interaction is due to the deprotonation of $\mu$-hydroxide ( $\mathrm{pH} 8.0$ ) to $\mu$-oxide $(\mathrm{pH} 10.0){ }^{37}$ When phosphate binds at $\mathrm{pH} 8.0$, an increase in ferromagnetic coupling is observed (from $\sim 1 \mathrm{~cm}^{-1}$ to $\sim 5 \mathrm{~cm}^{-1}$; Table 2). The magnitude of the coupling is similar for both wild type GpdQ (with $\mu-\mathrm{OH}$ intact) and the Asn80Asp mutant (without an intact $\mu-\mathrm{OH}$ ). Assuming that the coordination geometries are similar for the wild type and Asn80Asp enzyme, this may indicate that the exchange interaction occurs primarily through the $\mu-1,1$ aspartate ligand $(\mu-1,3$ bound phosphate does not need to be considered as a mediator of coupling). Most important influences on the magnitude of the coupling in dimetal complexes are the $\mathrm{M}-\mathrm{L}-\mathrm{L}$ bond angle and/or the sum of the $\mathrm{M}-\mathrm{L}$ bond distances. ${ }^{32}$ It is therefore likely that the increase in $J$ subsequent to phosphate binding is associated with an improved orbital overlap rather than a change in species through which the coupling is mediated. No change in the MCD spectra or VTVH MCD behavior is seen by adding phosphate at $\mathrm{pH} 10.0$. The $K_{\mathrm{i}}$ of phosphate at this $\mathrm{pH}$ is much larger than at $\mathrm{pH} 8^{8}$ and therefore the percentage of phosphate bound species will be insignificant. Thus, the observed $\sim 10$-fold increase in coupling from $\mathrm{pH} 8.0$ to 10.0 (from $\sim 1 \mathrm{~cm}^{-1}$ to $\sim 10 \mathrm{~cm}^{-1}$; Table 2) is consistent with the deprotonation of the $\mu$-hydroxide.

Conclusions. Using MCD spectra, ligand field calculations, and electronic structural parameters obtained by VTVH MCD analysis, the analysis presented here substantiates the previous interpretation ${ }^{8}$ of the MCD bands observed in wild type GpdQ as arising from a mixed sixand five-coordinate dinuclear center. The results also support the proposed mechanism which leads to a product bound structure in which phosphate is bound to both metal ions (perturbing the MCD transitions in both the $\alpha$ and $\beta$ cobalt ions), and in which the $\mu$-hydroxide acts as an activator of the nucleophile (a hydroxide bound

(37) Note that a strongly coupled $\mu$-oxo bridged metal ion center is observed in a $\mathrm{Fe}$ (III)-Mn(II) purple acid phosphatase from sweet potato at a $\mathrm{pH} \leq 5.0$. The low $\mathrm{p} K_{\mathrm{a}}$ for the $\mu$-hydroxide is due to the increased positive charge and hydrogen bonding interactions. Schenk, G.; Gahan, L. R.; Carrington, L. E.; Mitić, N.; Valizadeh, M.; Hamilton, S. E.; de Jersey, J.; Guddat, L. W. Proc. Natl. Acad. Sci. U.S.A. 2005, 102, 273-278.

(38) Paulat, F.; Lehnert, N. Inorg. Chem. 2008, 47, 4963-4976. terminally to the $\mathrm{Co}(\mathrm{II})$ in the $\alpha$ binding site; Figure 1), but itself is not a nucleophile. In wild-type GpdQ a hydrogen bonding interaction between the $\mu$-hydroxide and the terminal one activates the latter for the nucleophilic attack. However, in the Asn80Asp mutant of this enzyme, the strong coordination bond between the metal ion and the aspartate ligand leads to a rupture of the bond between the $\mu$-hydroxide and that metal ion. This perturbs the hydrogen bonding interaction between the two hydroxides, consistent with a nearly 10 -fold drop in reactivity measured for the mutant. Hence, in summary the analysis of the electronic structure of the dicobalt(II) center in GpdQ has led to the substantiation of (i) a terminally bound nucleophile, (ii) an activation mechanism for this nucleophile via hydrogen bonding interaction with the $\mu$-hydroxide, and (iii) a $\mu-1,3$ bridging mode of the phosphate group in the product stage of the catalytic cycle. This study is the first which examines the electronic structure of an enzyme with a $\mu-1,1$-carboxylate bridged dicobalt center and, in conjunction with a recently reported model complex ${ }^{29}$ suggests that these ligands can have a dominant effect on the electronic exchange interaction and give rise to ferromagnetic coupling.

Acknowledgment. J.A.L. wishes to acknowledge the National Science Foundation (U.S.A.) for financial support from Grant CHE0848433. G.S., D.L.O., and L.R.G. acknowledge funding from the Australian Research Council.

\section{Abbreviations}

\section{Glossary}

AOM angular overlap model

GpdQ glycerophosphodiesterase (from Enterobacter aerogenes)

MCD magnetic circular dichroism

VTVH variable temperature, variable field

ZFS zero field splitting

Supporting Information Available: Figures S1 and S2, Table $\mathrm{S} 1$, and equation $\mathrm{S} 1$. This material is available free of charge via the Internet at http://pubs.acs.org. 